\title{
Postoperative Atrial Fibrillation Understanding Causes and Risks
}

\author{
Marcel de Paula Pereira ${ }^{1 *}$ and Matheus de Paula Pereira ${ }^{2}$ \\ ${ }^{1}$ Heart Institute, University of São Paulo, Brazil \\ ${ }^{2}$ Universidade de Franca, Brazil
}

*Corresponding author: Marcel de Paula Pereira, Heart Institute of University of São

Paulo, São Paulo, Brazil.

\begin{abstract}
Atrial fibrillation (AF) is a common complication after surgery. It is reported to occur in 25-40\% of patients who undergo cardiac surgery [1] and it is less common in non-cardiac surgery. Postoperative atrial fibrillation (POAF) increases the risk of stroke, hospitalization, morbidity and mortality in 30 days. In this article, we review the mechanism, risk factors, prophylactic medications, complications after POAF and management.
\end{abstract}

Keywords: Atrial fibrillation; Postoperative atrial fibrillation; Cardiac surgery; Non-cardiac surgery

Abbreviations: AF: Atrial Fibrillation; POAF: Postoperative Atrial Fibrillation; CABG: Coronary Artery Bypass

\section{Introduction}

Atrial fibrillation (AF) is a common complication after surgery. It is reported to occur in $25-40 \%$ of patients who undergo cardiac surgery [1-3] and it is less common in non-cardiac surgery ones [4,5]. POAF is associated with increased length of hospital stay, higher rates of complications and mortality. Strategies to reduce POAF has been tested with beta-blockers and amiodarone diminishing the incidence of POAF. When POAF occurs, the treatment is mainly based on studies of patients undergoing cardiac surgery, with less evidence in the non-cardiac surgery. The strategies of rate or rhythm control has been tested in a randomized trial [2], and the aim of rhythm control therapy is to improve AF-related symptoms in POAF. Observational trials show that POAF is associated with an increased early stroke risk, increased morbidity and short and long term mortality [3, 5-7], after cardiac and non-cardiac surgery. Additional data is needed to determine the benefits and risks fo long-term anticoagulation and whether or not acute postoperative $\mathrm{AF}$ ( $<48$ hours) carry similar risks in comparison to long term $\mathrm{AF}$ $(>48$ hours)

\section{Discussion}

$\mathrm{AF}$ is the most common arrhythmia treated in clinical practice and themostcommonarrhythmia for which patientsare hospitalized. In addition, about 33\% of arrhythmia-related hospitalizations are for AF [1]; AF is associated with approximately a fivefold increase in the risk of cerebrovascular accident (stroke) and a twofold increase in the risk of all-cause mortality. AF is a common complication after open cardiac surgery, with valve and coronary artery bypass (CABG) being the most reported cases (25-40\%). In non-cardiac surgery, POAF usually occurs in surgeries like large esophageal procedures (esophagectomy) or large pulmonary surgeries (pneumonectomy, lung transplantation or resection of anterior mass). The incidence is about 15\% [4]. The peak of incidence of POAF is on the second day postoperative. The pathogenesis is multifactorial and probably involves adrenergic activation, inflammation, atrial ischemia, electrolyte disturbances, hypoxia and genetic factors. Several risk factors for AF after open heart surgery have been identified, including age over 70 years, history of prior AF, male gender, left ventricular dysfunction, left atrial enlargement, chronic lung disease, diabetes, and obesity [1]. Beta-blockers reduce POAF [8] and supraventricular tachycardia and it is recommended for the prevention in ESC guidelines [9]. Amiodarone reduce the incidence of POAF [10] and should be considered as prophylactic therapy to prevent AF after cardiac surgery [9]. There are smalls studies with other therapies, but have not demonstrated clear beneficial effects. This include magnesium, colchicine and corticosteroids [9]. When POAF occurs, multifactorial factors are involved. Postoperative 
complications (cardiac tamponade, mechanical complications or others complications), patient pain, electrolyte disturbances, hypoxia or infection are common causes and should be treated. When patients are haemodynamically unstable, cardioversion and consideration of antiarrhythmic drugs is reccomended, preferably amiodarone or vernakalant. In haemodynamically stable patients, rhythm or rate control can be done. In a randomized comparison of rate and rhythm-control strategies in patients with AF after cardiac surgery [2], there were no significant differences between the two strategies in the number of days of hospitalization, mortality, or adverse events. In 60 days, $95 \%$ of new onset $\mathrm{AF}$ was in sinus rhythm and not experienced AF during the prior 30 days. Treatment with an oral anticoagulant should be continued after discharge. Because new-onset AF after cardiac surgery often does not recur after 60 to 90 days, rhythm-control medications can be discontinued at that time, and if there is no subsequent evidence of symptomatic or asymptomatic $\mathrm{AF}$, as confirmed by monitoring (e.g., 3- to 4-week auto trigger event monitor), anticoagulation can be safely discontinued unless needed for another indication [1]. Good quality data are needed to determine if long-term anticoagulation can prevent strokes in patients with postoperative AF or in acute episodes of $\mathrm{AF}$ ( $<48$ hours).

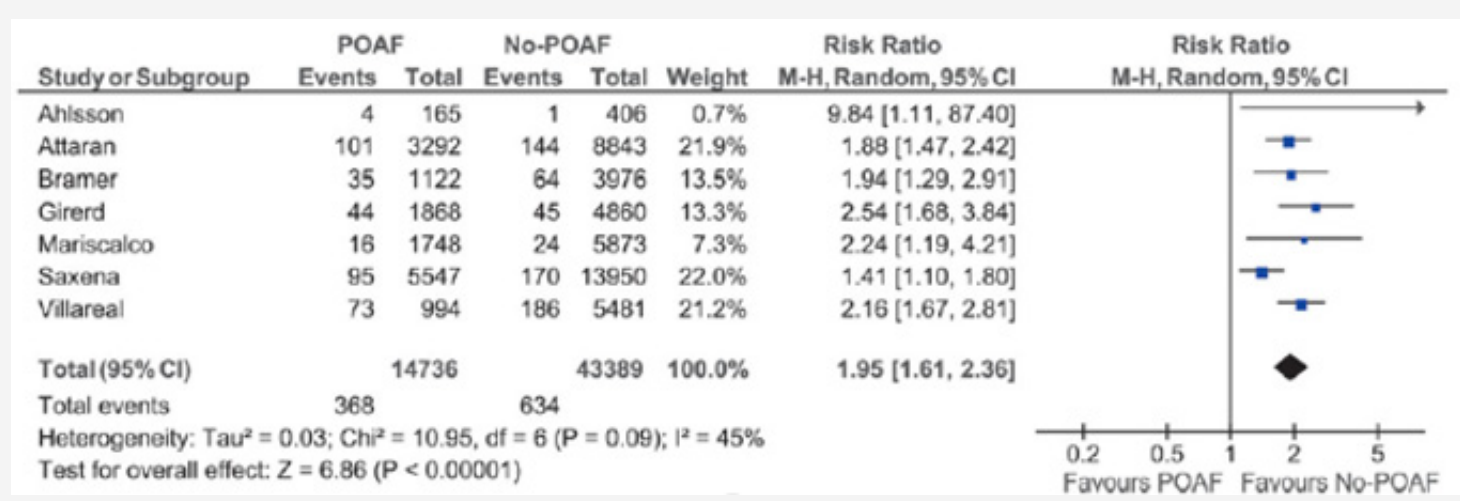

Figure 1: Forest plot of 30-day mortality risk associated with POAF following CABG.

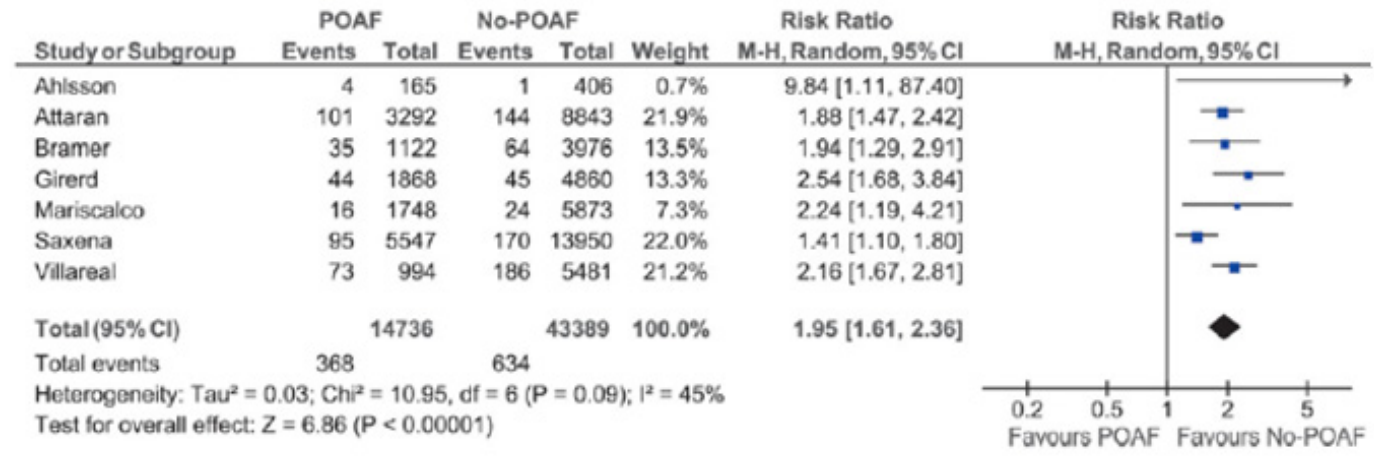

Figure 2: Forest plot of stroke in POAF versus no POAF.

\begin{tabular}{|c|c|c|c|c|c|c|c|}
\hline Study or Subgroup & log[Hazard Ratio] & SE & $\begin{array}{l}\text { POAF } \\
\text { Total }\end{array}$ & $\begin{array}{r}\text { No-POAF } \\
\text { Total } \\
\end{array}$ & Weight & $\begin{array}{l}\text { Hazard Ratio } \\
\text { IV, Random, } 95 \% \mathrm{Cl}\end{array}$ & $\begin{array}{l}\text { Hazard Ratio } \\
\text { IV, Random, } 95 \% \mathrm{Cl}\end{array}$ \\
\hline Ahlsson & 0.444686 & 0.121638 & 165 & 406 & $5.1 \%$ & $1.56[1.23,1.98]$ & \\
\hline Al-Shaar & 0.24686 & 0.00397 & 1211 & 5094 & $12.9 \%$ & $1.28[1.27,1.29]$ & * \\
\hline Bramer & 0.307485 & 0.119816 & 1122 & 3976 & $5.2 \%$ & $1.36[1.08,1.72]$ & \\
\hline El-Chami & 0.19062 & 0.044394 & 2985 & 13184 & $10.8 \%$ & $1.21[1.11,1.32]$ & \\
\hline Filardo & 0.254642 & 0.05965374 & 1814 & 5085 & $9.5 \%$ & $1.29[1.15,1.45]$ & \\
\hline Girerd & 0.30010459 & 0.149603 & 1868 & 4860 & $3.9 \%$ & $1.35[1.01,1.81]$ & \\
\hline Mariscalco & 0.19885086 & 0.059163 & 1748 & 5873 & $9.5 \%$ & $1.22[1.09,1.37]$ & \\
\hline ONeal & 0.09531018 & 0.009193 & 2537 & 13165 & $12.8 \%$ & $1.10[1.08,1.12]$ & $\cdot$ \\
\hline ONeal2 & 0.33647224 & 0.068128 & 370 & 13165 & $8.8 \%$ & $1.40[1.23,1.60]$ & \\
\hline Saxena & 0.17395331 & 0.052897 & 5547 & 13950 & $10.0 \%$ & $1.19[1.07,1.32]$ & \\
\hline Thoren & 0.33647224 & 0.0352 & 2152 & 4669 & $11.5 \%$ & $1.40[1.31,1.50]$ & $\rightarrow$ \\
\hline Total $(95 \% \mathrm{Cl})$ & & & 21519 & 83427 & $100.0 \%$ & $1.28[1.19,1.37]$ & \\
\hline \multicolumn{7}{|c|}{$\begin{array}{l}\text { Heterogeneity: } \text { Tau }^{2}=0.01 ; \mathrm{Chi}^{2}=247.98, \mathrm{df}=10(P<0.00001) ; \mathrm{I}^{2}=96 \% \\
\text { Test for overall effect: } Z=6.87(P<0.00001)\end{array}$} & $\begin{array}{c}0.70 .8511 .21 \\
\text { ours POAF Favours }\end{array}$ \\
\hline
\end{tabular}

Figure 3: Forest plot of overall long-term mortality risk associated with POAF following CABG. 
POAF is associated with an increased early stroke risk, increased morbidity and 30-day mortality [3, 5-7]. In a retrospective registry [3], Management of Postoperative Atrial Fibrillation and subsequent outcomes in contemporary patients undergoing cardiac surgery: Insights from the Society of thoracic surgeons CAPS-CARE Atrial Fibrillation registry, 2390 patients who undergo to $\mathrm{CABG}, 28 \%$ developed POAF. According to a univariate analyses, POAF increase 30 day mortality and stroke. In a meta-analyses [7], stroke and mortality were higher in patients with POAF in 30 days. Hospitalization and long-term mortality was higher in patients with POAF (Figures 1-3).

In a registry of non-cardiac surgeries, patients who developed POAF had similar long-term risk of thromboembolism when compared to patients who had previous non-valvular $\mathrm{AF}$, in a median follow-up of 10 years.

\section{Conclusion}

Atrial Fibrillation is the most common arrhythmia in clinical practice. POAF is a common complication and carries high risk of stroke, morbidity and mortality in 30 days and long-term. Prophylactic Beta-Blockers or Amiodarone should be initiated before surgery in high risk patients. The strategy of rhythm or rate control are similar in stable patietns with new-onset $\mathrm{AF}$ and rhythm control are preferred in unstable patients. Anticoagulation are indefinitely at least for 60-90 days or indefinetelly in new-onset AF after surgery. Although best evidence is based in arrhythmias following cardiac surgery, the same recommendations are used in POAF following non-cardiac surgery.

\section{Acknowledgments}

None.

\section{Conflict of Interest}

No conflict of interest.

\section{References}

1. (2018) Braunwald's Heart Disease Review and Assessment. (11 $1^{\text {th }}$ edn.), pp. 730-744.

2. Gillinov AM, Bagiella E, Moskowitz AJ, Raiten JM, Groh MA, et al. (2016) Rate Control versus Rhythm Control for Atrial Fibrillation after Cardiac Surgery. N Engl J Med 374: 1911-1921.

3. Steinberg BA, Zhao Y, He X, Hernandez AF, Fullerton DA, et al. (2014) Management of Postoperative Atrial Fibrillation and Subsequent Outcomes in Contemporary Patients Undergoing Cardiac Surgery: Insights From the Society of Thoracic Surgeons CAPS-Care Atrial Fibrillation Registry. Clin Cardiol 37(1): 7-13.

4. Frendl G, Sodickson AC, Chung MK, Waldo AL, Gersh BJ, et al. (2014) AATS guidelines for the prevention and management of perioperative atrial fibrillation and flutter for thoracic surgical procedures. The Journal of Thoracic and Cardiovascular Surgery 148(3): e153-e193.

5. Butt JH, Olesen JB, Havers-Borgersen E, Gundlund A, Andersson C, et al. (2018) Risk of Thromboembolism Associated With Atrial Fibrillation Following Noncardiac Surgery. Journal of the American College of Cardiology 72(17):2027-2036.

6. Gialdini G, Nearing K, Bhave PD, Bonuccelli U, Iadecola C, et al. (2014) Perioperative Atrial Fibrillation and the Long-term Risk of Ischemic Stroke. JAMA 312(6): 616-622.

7. Phan K, Ha HS, Phan S, Medi C, Thomas SP, et al. (2015) New-onset atrial fibrillation following coronary bypass surgery predicts long-term mortality: a systematic review and meta-analysis. European Journal of Cardio-Thoracic Surgery 48(6): 817-824.

8. Khan MF, Wendel CS, Movahed MR (2013) Prevention of post-coronary artery by-pass grafting (CABG) atrial fibrillation: efficacy of prophylactic beta-blockers in the modern era: a meta-analysis of latest randomized controlled trials. Ann Noninvasive Electrocardiol 18: 58-68.

9. Kirchhof P, Benussi S, Kotecha D, Ahlsson A, Atar D, et al. (2016) ESC Guidelines for the management of atrial fibrillation; Developed in collaboration with EACTS. European Heart Journal 37: 2893-2962.

10. Chatterjee S, Sardar P, Mukherjee D, Lichstein E, Aikat S, et al. (2013) Timing and route of amiodarone for prevention of postoperative atrial fibrillation after cardiac surgery: a network regression meta-analysis. Pacing Clin Electrophysiol 36(8): 1017-1023. 